\title{
Três Décadas de MAPA - Monitorização Ambulatorial da Pressão Arterial de 24 horas. Mudanças de Paradigmas no Diagnóstico e Tratamento da Hipertensão Arterial
}

\author{
Fernando Nobre, Eduardo Barbosa Coelho \\ Ribeirão Preto, SP
}

Desde que Riva-Rocci, em 1886, criou o esfigmomanômetro ${ }^{1}$, as medidas de pressão casuais têm sido utilizadas para a avaliação da pressão arterial, determinando o diagnóstico, estabelecendo prognóstico e avaliando a eficácia do tratamento anti-hipertensivo instituído.

Nas últimas três décadas, entretanto, muito tem sido questionado sobre o valor dessas medidas. Sabe-se, por exemplo, que significativa percentagem de pacientes apresenta um valor de pressão arterial muito diverso, quando examinados na clínica pelo médico ou fora dela, pela enfermeira ou por eles próprios ${ }^{2,3}$. Isso pode resultar em falsa e, conseqüentemente errônea, interpretação do comportamento da pressão arterial, levando, entre outras coisas, a diagnóstico incorreto e, por via de conseqüência, a condutas inapropriadas.

Está sendo observada uma rápida e consistente mudança de paradigmas com relação ao melhor modo de se avaliar o comportamento da pressão arterial com os mais diversos objetivos. Nesse cenário, o método que mais se consolida é, sem dúvidas, a monitorização ambulatorial da pressão arterial-MAPA, além das medidas residenciais da pressão arterial-MRPA.

Isso se deve, principalmente, ao avanço no conhecimento das técnicas de avaliação da pressão arterial em $24 \mathrm{~h}$; à utilização de equipamentos de novas gerações com características mais apropriadas, fáceis de serem usados, com custos cada dia mais acessíveis; validados por protocolos internacionais rigorosos, totalmente automáticos e eletronicamente sofisticados, que conferem grande confiabilidade ao seu desempenho $0^{4,5}$.

As evidências de que as medidas de pressão obtidas

Faculdade de Medicina de Ribeirão Preto USP

Correspondência: Fernando Nobre - Av. Independência, 3767 - 14026-150 Ribeirão Preto, SP - e-mail:fernando.nobre@uol.com.br

Recebido para publicação em 4/11/02

Aceito em 24/3/03 pela MAPA são melhor correlacionadas com os riscos decorrentes da hipertensão arterial, quando comparadas com as medidas convencionais, constituem-se em outro fator decisivo para a sua crescente utilização ${ }^{6}$.

Em 1964, Herbert e cols. ${ }^{7}$ demonstraram benefícios da MAPA e a possibilidade atraente de avaliar a pressão durante as atividades usuais do paciente. Nessa época os equipamentos utilizados eram semi-automáticos, desconfortáveis e extremamente ruidosos, constituindo-se essas características importantes limitações ao seu rotineiro uso.

Um excelente indicador da crescente utilização da MAPA e da consistência de conhecimentos a seu respeito, pode ser observado quando se analisa, cronologicamente, as publicações sobre o método e suas aplicabilidades. Assim, até 1989 identificam-se 137 trabalhos publicados, em periódicos científicos indexados, sobre MAPA, enquanto que de 1990 até os dias atuais esse número é representado por 2855 publicações.

A produção de inúmeras diretrizes para o uso da MAPA, normatizando sua utilização e, conseqüentemente, fazendo seu uso racional e cientificamente correto, tem contribuído para sua utilização cada vez mais consistente e ampliada. Data da década de 1960 a primeira publicação demonstrando as possibilidades de avaliação da pressão em $24 \mathrm{~h}^{8}$. As indicações, baseadas nessas crescentes investigações, estão claramente definidas e explicitadas na tabela I, de conformidade com as mais importantes diretrizes para o uso da MAPA.

As limitações para o uso do método, igualmente segundo os principais documentos orientadores para seu uso, estão expressas no quadro I, cabendo ressaltar que algumas delas são hoje menos expressivas que no passado, em decorrência da evolução tecnológica que os aparelhos experimentaram e do aprimoramento sobre a prática da MAPA em si.

Assim, é lícito afirmar-se que, pelos vários fatores citados, há um crescente uso da MAPA, representado pelo conhecimento de que os dados obtidos por seu intermédio 


\begin{tabular}{|c|c|c|c|c|c|c|c|}
\hline & GHL & III Diretrizes & JNC VI & SHA & FSH & ASH & Canadian \\
\hline HAB & $\mathrm{S}$ & $\mathrm{S}$ & $\mathrm{S}$ & $\mathrm{S}$ & $\mathrm{S}$ & $\mathrm{S}$ & $\mathrm{S}$ \\
\hline HAS grave & $\mathrm{N}$ & $\mathrm{N}$ & $\mathrm{N}$ & $\mathrm{N}$ & $\mathrm{S}$ & $\mathrm{N}$ & $\mathrm{N}$ \\
\hline HAS episódica & $\mathrm{S}$ & $\mathrm{S}$ & $\mathrm{S}$ & $\mathrm{N}$ & $\mathrm{S}$ & $\mathrm{S}$ & $\mathrm{S}$ \\
\hline HAS secundária & $\mathrm{S}$ & $\mathrm{N}$ & $\mathrm{S}$ & $\mathrm{N}$ & $\mathrm{N}$ & $\mathrm{N}$ & $\mathrm{N}$ \\
\hline HAS - gravidez & $\mathrm{N}$ & $\mathrm{N}$ & $\mathrm{N}$ & $\mathrm{S}$ & $\mathrm{S}$ & $\mathrm{N}$ & $\mathrm{S}$ \\
\hline Disautonomia & $\mathrm{N}$ & $\mathrm{s}$ & $\mathrm{S}$ & $\mathrm{s}$ & $\mathrm{S}$ & $\mathrm{s}$ & $\mathrm{S}$ \\
\hline HAS - diabetes & $\mathrm{N}$ & $\mathrm{N}$ & $\mathrm{N}$ & $\mathrm{S}$ & $\mathrm{N}$ & $\mathrm{N}$ & $\mathrm{N}$ \\
\hline
\end{tabular}

GHL - Germany Hypertension League; III Diretrizes - III Diretrizes Brasileiras para uso da MAPA; JNC VI - Joint National Committee; SHA-Swiss Hypertension Association; FSH - French Society of Hypertension; ASH - American Society of Hypertension; Canadian - Consenso Canadense para uso da MAPA; HÁ Hipertensão do Avental Branco; HAS - Hipertensão Arterial Sistêmica.

\begin{tabular}{|l|}
\hline Quadro I - Principais limitações para o uso da MAPA \\
\hline Relacionadas aos equipamentos \\
- Não validação de monitores \\
- Perdas de dados \\
- Desconforto no sono e no trabalho \\
Falta de critérios de normalidade populacionais \\
Custo alto \\
\hline
\end{tabular}

melhor expressam o comportamento da pressão arterial, pelo desenvolvimento de equipamentos mais confortáveis, seguros e confiáveis e, consequentemente, pela redução significativa das limitações para o seu uso rotineiro.

Esse crescente uso na prática clínica, deverá ser muito ampliado em decorrência do fato de que os seguros de saúde em todo o mundo, provavelmente estimulados por esses dados, estão incorporando a MAPA no elenco de exames reconhecidos como úteis e aceitos para realização e pagamento.

É, pois, imperativo e oportuno que se avalie, à luz dos conhecimentos atuais, as indicações gerais e pontuais, seus benefícios, limitações e aplicabilidade nas mais diversas situações, da monitorização ambulatorial da pressão arterial de $24 \mathrm{~h}$.

Por razões didáticas, faremos a análise das suas utilidades em três contextos específicos: como coadjuvante no diagnóstico da hipertensão arterial, no estabelecimento do prognóstico dos hipertensos e na avaliação da eficácia terapêutica anti-hipertensiva em uso.

\section{MAPA e sua contribuição para o diagnóstico da hipertensão}

O uso da MAPA para o diagnóstico da hipertensão arterial tem sido largamente ampliado e vem sendo referendado por diretrizes como o VI Joint National Committee $(\mathrm{JNC} \mathrm{VI})^{9}$ nos Estados Unidos, pelo documento endossado pela International Society of Hypertension (ISH) e World Health Organization $(\mathrm{WHO})^{10} \mathrm{e}$ pelas IV Diretrizes Brasileiras para o Tratamento da Hipertensão Arterial ${ }^{11}$.

De um modo geral, o objetivo principal está centrado na decisão entre tratar ou não um determinado paciente com base nos valores obtidos de pressão arterial.
Embora tenha havido, nos últimos tempos, forte decisão de não se levar em consideração apenas os níveis de pressão arterial a fim de se instituir o tratamento anti-hipertensivo, é inquestionável que a obtenção de valores de pressão confiáveis e realmente representativos do comportamento da pressão arterial é indispensável.

Recente determinação, emanada em Outubro de 2001, dos Centers for Medicare and Medcaid Services, recomendou que a MAPA deveria ser paga para os pacientes com suspeita de hipertensão do avental branco, caracterizada por níveis de pressão acima de 140 x $90 \mathrm{~mm} \mathrm{Hg}$ em pelo menos três visitas médicas e valores inferiores em duas ocasiões fora do consultório e sem lesões de órgãos-alvo ${ }^{12}$.

Recentemente ${ }^{13}$ reconheceu-se a importância do efeito do avental branco no diagnóstico e no controle da hipertensão arterial no cuidado primário. Nesse estudo os autores afirmam que não é mais tempo de utilizar-se apenas os valores de pressão obtidos pelo médico em geral para tomar a decisão de se instituir o tratamento anti-hipertensivo.

Esse fato representa um reconhecimento do papel da MAPA para essa circunstância específica, altamente prevalente e de grande impacto social e econômico.

Valores maiores de pressão arterial na presença do médico em relação àqueles obtidos fora do consultório caracterizam o efeito do avental branco.

Se a sua magnitude faz com que haja mudança de diagnóstico, configurando hipertensão no consultório sem correspondência fora dele, firma-se o diagnóstico de hipertensão do avental branco.

Mais recentemente, se identificou uma população que apresenta comportamento oposto. São indivíduos com pressão normal no consultório e alterada na MAPA. Para esse tipo de comportamento, definido como normotensão do avental branco, ainda não se tem explicação consensualmente aceitável, não se conhecendo seus valores clínicos e prognósticos exatos. A figura 1 expressa muito bem as várias possibilidades decorrentes das diferenças de pressão arterial obtidas no consultório e por intermédio da MAPA.

Três pontos são fundamentais para ser avaliados em relação à hipertensão do avental branco: $1^{\circ}$ ) a sua ocorrência não parece ser tão benigna quanto se admitiu em passado recente, havendo evidências no sentido de que os indivíduos nessa situação comportam-se de modo peculiar, com risco cardiovascular provavelmente intermediário entre hi- 


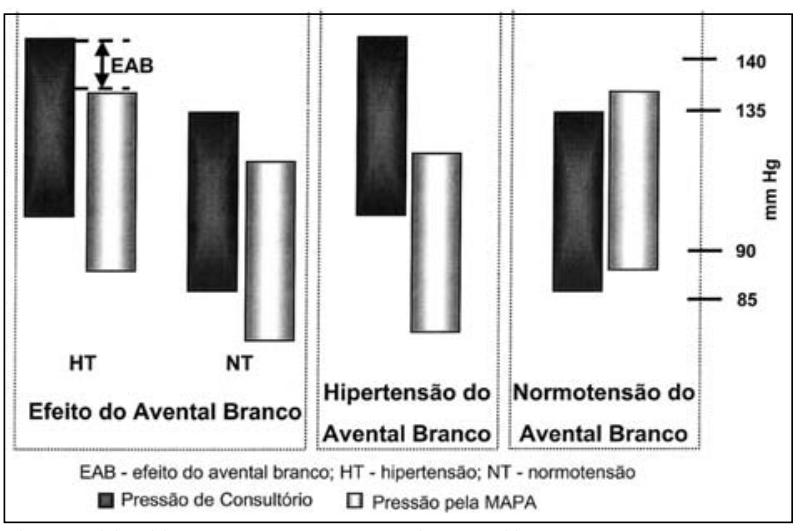

Fig. 1 - Efeito, hipertensão e normotensão do avental branco

pertensos e normotensos ${ }^{14-16} ; 2^{\circ}$ ) que esses pacientes necessitam de seguimento e, em casos específicos, de tratamento medicamentoso, sendo as mudanças de hábitos de vida imperativas para todos; $3^{\circ}$ ) os hipertensos do avental branco têm maior chance de se tornarem hipertensos estabelecidos ${ }^{17,18}$. Assim, constitui-se consenso que esses indivíduos necessitam de seguimento havendo recomendação para realização anual de MAPA $^{19}$.

No algoritmo apresentado na figura 2, indicamos os aspectos relacionados ao diagnóstico, tratamento e seguimento dos pacientes com hipertensão do avental branco.

Algumas condições menos freqüentes, porém igualmente relacionadas ao diagnóstico de hipertensão, poderão, ainda, constituir-se em indicações para a MAPA. Dentre elas, apontamos a hipertensão episódica ou paroxística e a hipertensão limítrofe. A hipertensão episódica tem a sua mais clássica manifestação afeita ao quadro de feocromocitoma, podendo, ou não, ser identificada pela MAPA. Quanto à hipertensão limítrofe essa é uma formal indicação da maioria das diretrizes (tab. I).

Mas, resta-nos, ainda, uma questão de ordem prática não absolutamente respondida: a despeito de todas as considerações sobre o método de avaliação da pressão arterial nas 24h, como, na prática clínica, aplicar, com parcimônia, a MAPA ?

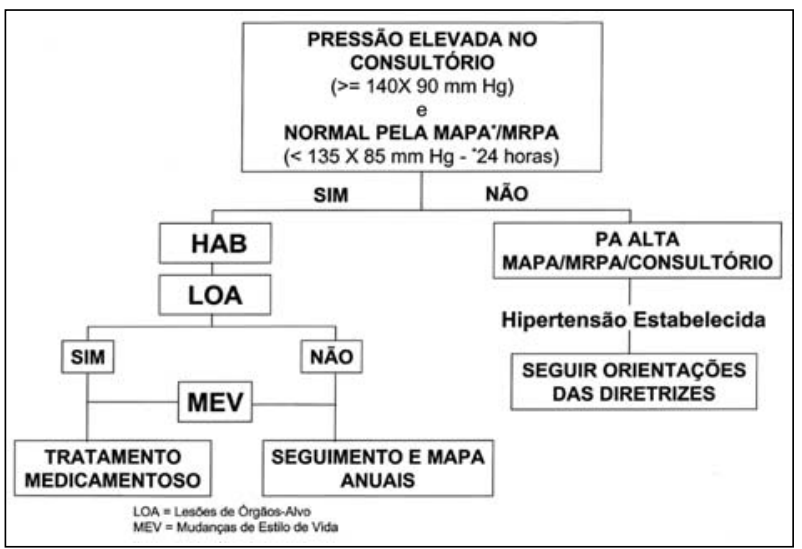

Fig. 2 - Algoritmo para diagnóstico, tratamento e seguimento da hipertensão do avental branco (HAB).
Para responder essa indagação podemos recorrer à recente investigação de Stergiou e cols. ${ }^{20}$ que avaliaram 106 pacientes, sabidamente hipertensos, submetidos às medidas casuais - de consultório, à MAPA e a medidas domiciliares, encontrando praticamente a mesma sensibilidade diagnóstica para os três métodos utilizados: $70 \%$ para as medidas de consultório, $58 \%$ para a MRPA e $62 \%$ para a MAPA. A identificação de hipertensão do avental branco, uma entidade com prevalência próxima de 20 a $30 \%$ entre os hipertensos leves a moderados, só pode ser, entretanto, diagnosticada com o uso da MAPA, constituindo-se esse um importante diferencial do método.

A fim de atender a essa questão, Pickering ${ }^{21}$ propôs um fluxograma, com fins diagnósticos, para a utilização racional de cada um dos métodos como mostrado na figura 3 , modificada por nós.

Seria, portanto, correto dizer-se, após essas reflexões, que a MAPA está inquestionavelmente indicada na suspeita de hipertensão do avental branco, onde sua aplicação é formalmente definida nas diretrizes que normatizam o seu uso e naquelas condições em que haja dúvidas quanto ao real comportamento da pressão arterial pelas medidas casuais.

\section{MAPA e prognóstico do paciente hipertenso}

Medidas de pressão arterial - Há uma bem demonstrada melhor correlação entre lesão em órgãos-alvo e os valores de pressão arterial obtidos pela MAPA do que aqueles decorrentes das medidas casuais ${ }^{22-24}$.

Perloff e cols. ${ }^{25,26}$ demonstraram, ainda na década de 80 , o valor prognóstico das médias de pressão de vigília obtidas pela MAPA.

Okubo e cols. ${ }^{27}$ estabeleceram correlação entre níveis de pressão arterial pela MAPA e mortalidade. Definiram valores médios de pressão arterial sistólica de 128 (120-133) mmHg e 74 (65-78)mmHg para a diastólica, a partir dos quais havia evidente e crescente aumento de mortalidade. A despeito dessa investigação ser particularizada a uma população idosa de japoneses e, portanto, com características peculiares, demonstrou-se o poder de correlação entre níveis de pressão arterial, pela MAPA, e prognóstico através desse estudo muito bem delineado e com tempo de seguimento apropriado.

No Systolic Hypertension in Europe (Syst-Eur) $S t u d y^{28}$, igualmente demonstrou-se, pela MAPA, em 808 pacientes avaliados, em período de seguimento médio de 4,4 anos, que os valores de pressão obtidos, particularmente a média de pressão sistólica durante o sono, foram significantes preditores de eventos totais, cardíacos e cerebrovasculares.

Assim, acumulam-se evidências, de que as pressões obtidas pela MAPA oferece uma consistente correlação com prognóstico e, se comparadas com as medidas casuais, de longe são mais fortes preditores de risco total, cardíaco e cerebrovascular. 
Ritmo vigília-sono - A pressão arterial durante o sono, somente pode ser acessada pela MAPA. Oferece uma singular possibilidade de análise do comportamento entre os períodos de vigília e sono, nas $24 \mathrm{~h}$.

Há evidências de que o comportamento da pressão nas $24 \mathrm{~h}$, levando-se em consideração os dois períodos em questão, representa importante aspecto em relação ao prognóstico.

O’Brein e cols. ${ }^{29}$, em 1988, em carta ao periódico Lancet, sugeriram que os pacientes cuja pressão arterial não apresentava uma redução de pelo menos $10 \%$ entre os períodos de vigília e sono apresentavam maior probabilidade de acidentes encefálicos.

Várias investigações estabeleceram, posteriormente, a maior probabilidade de lesões em órgãos-alvo nas populações hipertensas que não apresentam, no mínimo, $10 \%$ de queda entre os períodos de vigília e sono.

Assim, maior Índice de massa do ventrículo esquer$\mathrm{do}^{30}$, presença de microalbuminuria ${ }^{31}$ e maior probabilidade de lesões encefálicas ${ }^{32}$ foram correlacionadas com ausência de descenso entre os dois períodos em questão.

Variabilidade - Adespeito da importância da variabilidade da pressão arterial, como marcador prognóstico a avaliação desse parâmetro, é mal feita pela MAPA. Assim, não se pode inferir, pelo desvio-padrão que estima a variabilidade da pressão arterial pela MAPA, o prognóstico dos indivíduos avaliados.

Por outro lado, estudos utilizando medidas intra-arteriais $^{33}$ ou medidas não invasivas pela MAPA ${ }^{34}$ mostraram freqüência e gravidade de lesões em órgãos-alvo maiores nos indivíduos com maior variabilidade da pressão arterial.

No estudo PIUMA ${ }^{35}$ para um mesmo nível de pressão sistólica de 24h, os hipertensos foram classificados em duas categorias: acima e abaixo da média do grupo. A massa do ventrículo esquerdo,avaliada pela ecocardiografia,não foi diferente nos grupos acima e abaixo da média de variabilidade. Nesse mesmo estudo, os autores valeram-se do banco de dados obtido para avaliar o prognóstico de acordo com os diferentes níveis de variabilidade ultradiana da pressão arterial. Nessas condições, a taxa de eventos cardiovasculares maiores foi mais alta em indivíduos com desvio-padrão, representando a variabilidade, acima da média do grupo, comparado aos que tiveram menor desvio-padrão, que o total dos indivíduos estudados.Entretanto, essa diferença não se sustentou mediante uma análise multivariada após ajustada para idade, diabetes, eventos cardiovasculares prévios e medidas ambulatoriais da pressão arterial.

Em conclusão, cabe-nos afirmar que a variabilidade da pressão arterial, embora relacionada a prognóstico, particularmente com a utilização de métodos que permitam estimar a pressão arterial batimento-a-batimento, sofre influência de outros fatores, constituindo-se em um campo onde novos estudos ainda precisam ser conduzidos para definir o seu real papel, particularmente, utilizando-se dados obtidos pela MAPA.
Pressão de pulso - A pressão de pulso tem sido considerada um importante marcador prognóstico, particularmente, para os pacientes acima dos 55 anos $^{36}$.

Entretanto, cabe destacar que ela pode ser fortemente influenciada pela reação de alerta durante a visita médica, particularmente no que diz respeito à pressão arterial sistólica. Assim, a pressão de pulso estimada pelas medidas de consultório pode ser superestimada. Verdechia e cols. ${ }^{37}$ avaliaram 2010 pacientes, pela MAPA, e de acordo com a distribuição da pressão de pulso em três tercis, encontraram taxas de risco para eventos cardiovasculares totais, respectivamente de:1,19; 1,81 e 4,92 enquanto que para eventos fatais as taxas foram, também respectivamente, de: $0,11,0,17$ e 1,23 . Por esse estudo e para esse grupo de pacientes, os autores estratificaram como de alto risco os indivíduos que, pela MAPA, apresentaram pressão de pulso superior a $53 \mathrm{mmHg}$.

Ainda há necessidade de um estudo prospectivo de apropriado delineamento que possa determinar, pela MAPA, o real significado prognóstico da pressão de pulso na população em geral.

\section{MAPA e avaliação da terapêutica anti-hipertensiva}

É bem estabelecida a necessidade de adequado controle da pressão arterial nas $24 \mathrm{~h}$.

A avaliação e seguimento dos pacientes hipertensos sob tratamento medicamentoso pela MAPA, parece ser mais eficiente do que quando utilizadas as medidas de consultório.

Provavelmente, muitos dos pacientes que, a despeito de apresentarem medidas normais de pressão arterial no consultório, tiveram uma complicação grave decorrente de hipertensão, não exibiam pressão controlada durante as $24 \mathrm{~h}$.

A possibilidade de administração de medicamentos anti-hipertensivos a intervalos de tempo adequados, para um controle efetivo e continuado, da pressão arterial, só foi possível graças ao advento da monitorização ambulatorial da pressão arterial.

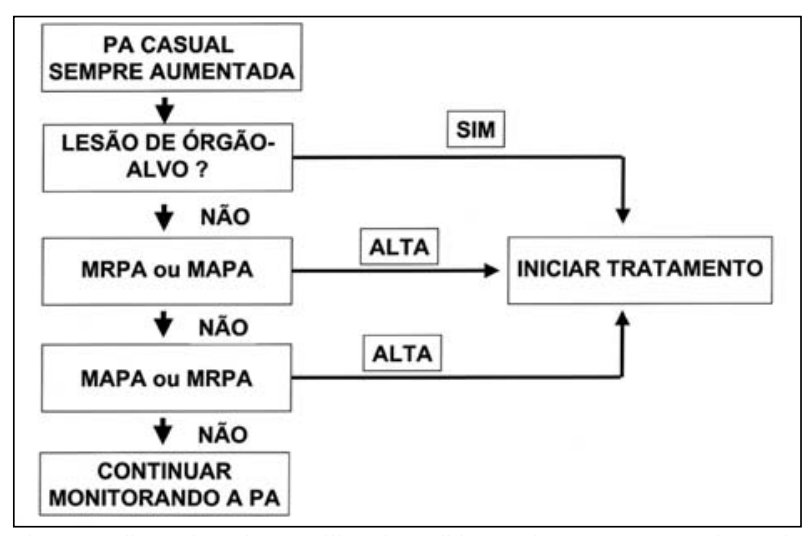

Fig. 3 - Avaliação de pacientes utilizando medida casual, MAPAe MRPA, de acordo com Pickering (21), modificado 
Por esse método pode-se estabelecer um coeficiente denominado trough:peak pelo qual os intervalos de administração dos anti-hipertensivos podem ser seguramente definidos.

Essa relação expressa o efeito anti-hipertensivo ainda presente imediatamente antes da administração da nova dose do medicamento testado (efeito trough) pelo máximo efeito observado (efeito peak) deduzidos os efeitos placebo em ambas as condições (fig. 4).

O FDA - Food and Drug Administration, nos Estados Unidos, definiu a necessidade de um valor mínimo de $50 \%$ para essa relação, para que a medicação anti-hipertensiva possa ser administrada em dose única diária.

Por essa importante contribuição oferecida pela MAPAé possível que estejamos nesse momento utilizando de forma mais racional e apropriada as medicações para o tratamento da hipertensão arterial.

O controle da pressão arterial avaliado pela MAPA, particularmente nos hipertensos tratados, é mais eficaz que pelas medidas de consultório. Subsidia essa afirmativa uma série de estudos que demonstraram, por exemplo, que o efeito de avental branco, comumente presente em hipertensos sob tratamento, é muito minimizado, senão abolido, quando a pressão é avaliada pela MAPA.

Entretanto, duas questões precisam ser consideradas. $1^{\circ}$ ) o custo para o controle dos hipertensos tratados, utilizando a MAPA não será muito mais elevado do que com a utilização das medidas de consultório? e, $2^{\circ}$ ) há evidências de que os indivíduos hipertensos tratados e controlados com base nas informações da MAPA terão melhor prognóstico, expressos por menores morbidade e mortalidade?

Em relação à $1^{\circ}$, Stäessen e cols..$^{38}$ demonstraram, em um estudo publicado em 1997, que em 419 pacientes hipertensos tratados, (213 tendo como base para o controle os dados da MAPA, comparados com 206 que valeram-se das medidas de consultório), não haver maior custo com o uso da MAPA durante o período de seguimento.Isso se deveu a três aspectos: os indivíduos com hipertensão do avental branco foram excluídos do grupo que recebeu tratamento medicamentoso; o número de medicamentos utilizados foi menor no grupo seguido pela MAPA e o número de consultas médicas foi também menor no grupo MAPA. Quando foram analisados os custos do seguimento em ambos os grupos, ainda que se considerando o valor da aplica-

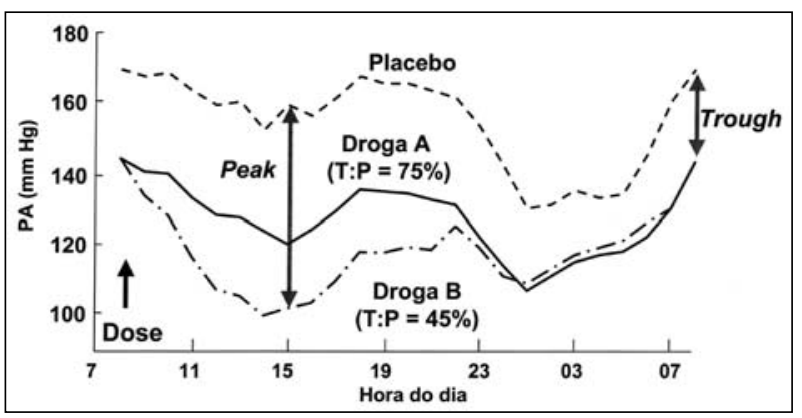

Fig. 4 - Relação T:P de duas drogas hipotéticas A e B. ção da MAPA repetidas vezes, isso foi compensado pelas duas outras circunstâncias anteriormente citadas.

Em relação à $2^{\circ}$ questão, Schrader e cols.$^{39}$ demonstraram, em estudo prospectivo e randomizado, envolvendo 851 pacientes, que a morbidade e mortalidade foram menores naqueles indivíduos que utilizaram os valores de pressão obtidos pela MAPA para orientar o tratamento anti-hipertensivo.

Nesse estudo, foram alocados 1298 pacientes dos quais 851 concluíram o seguimento de 5 anos, sendo que 439 tiveram as medidas de consultório como referência para o seguimento e 412 as obtidas pela MAPA. No grupo da MAPA foram registrados em 5 anos de seguimento, 20 eventos primários (morbidade e mortalidade totais e eventos cerebrovasculares) contra 35 no grupo de medidas de consultório $(p=0,037)$. Foram identificados $22 \%$ de hipertensos do avental branco, excluídos do tratamento anti-hipertensivo medicamentoso.

Com relação ao papel da MAPA na orientação do tratamento anti-hipertensivo, ainda são necessários estudos que, de forma incontestável, confirmem e ampliem as informações iniciais de que seu uso como referência resultará em menor morbidade e mortalidade decorrentes da hipertensão arterial.

Entretanto, a identificação dos indivíduos com hipertensão do avental branco é condição que já qualifica o uso parcimonioso do método.

\section{Evidências dos benefícios da MAPA na prática clínica}

Estudos que tiveram por objetivo estabelecer o papel da MAPA na prática clínica, foram recentemente avaliados em publicação de Pickering ${ }^{12}$ e estão explicitados na tabela II onde é possível uma visão cronológica da aplicação do método, nas diversas situações clínicas.

\section{Perspectivas}

Do mesmo modo que as medidas casuais de pressão começaram a ser aplicadas no final do século XIX, quando não se dominavam as técnicas, não se conheciam os critérios de normalidade e, sobretudo, não eram claros os benefícios da medida da pressão arterial, assim também comportou-se a MAPA no final do século XX e início do XXI.

Entretanto, se esforços não tivessem sido envidados para aprimorar-se o método de obtenção da medida esfigmomanométrica da pressão arterial, valores de referência não tivessem sido obtidos por amplos estudos epidemiológicos e, por conseguinte, sua aplicação não tivesse sido consolidada, não teríamos hoje sequer os conceitos básicos e fundamentais sobre o risco da pressão elevada e dos benefícios de seu adequado controle.

Assim devemos agir com relação à MAPA.

Somente a sua aplicação parcimoniosa, calcada em conclusões cientificamente aceitáveis à ampliação de seu uso, dar-nos-ão os subsídios necessários à utilização plena 


\begin{tabular}{|c|c|c|c|c|}
\hline Autor & Ano & Estudo & $\mathrm{n}$ & Conclusões \\
\hline Prisant & 1990 & Transversal & 55 & MAPA foi mais correlacionada a alterações ecocardiográficas que as medidas causais. \\
\hline Lemne & 1995 & Transversal & 161 & PAC correlacionou-se pouco com HVE contrariamente ao que ocorreu com a MAPA \\
\hline Lin & 1995 & Transversal & 171 & Severidade das complicações da HAS melhor correlacionada com PAS pela MAPA \\
\hline Staessen & 1997 & ECR & 419 & Pacientes tratados baseados na MAPA necessitaram de menos medicamentos \\
\hline Okubo & 1997 & Coorte & 1542 & Pressões pela MAPA relacionaram-se mais fortemente ã mortalidade que as casuais. \\
\hline Verdechia & 1997 & Série de Casos & 1522 & EAB não se correlacionou a eventos cardiovasculares. \\
\hline Imai & 1997 & Coorte & 1192 & $\begin{array}{l}\text { Grupo de MAPA mostrou diminuição de sobrevida nos mais altos quintis de PAS e } \\
\text { mais altos e baixos quintis de PAD }\end{array}$ \\
\hline Boley & 1997 & Transversal & 280 & $\begin{array}{l}\text { Hipertensos adultos não tratados MAPA em vigília e em casa mostrou forte relação } \\
\text { com HVE }\end{array}$ \\
\hline Palatini & 1997 & Coorte & 1095 & MAPA foi muito útil em identificar $\mathrm{HAB}$ \\
\hline Musialik & 1998 & Transversal & 30 & MAPA no sono correlacionou-se com HVE em idosos \\
\hline Redon & 1998 & Coorte & 86 & MAPA mostrou-se útil na estratificação de risco em hipertensos refratários. \\
\hline Staessen & 1999 & ECR & 808 & Em idosos PAS pela MAPA foi preditor independente de risco. \\
\hline Lantelme & 2000 & Transversal & 88 & $\begin{array}{l}\text { EAB e sua estimulação não são equivalentes. EAB não se mostrou relacionado a lesão de } \\
\text { órgãos-alvo. }\end{array}$ \\
\hline Schrader & 2000 & ECR & 851 & $\begin{array}{l}\text { Pacientes tratados tendo a MAPA com referência tiveram significativamente menos } \\
\text { morbidade e mortalidade toraos e eventos cerebrovasculares }\end{array}$ \\
\hline $\begin{array}{l}\text { MAPA - I } \\
\text { esquerda; } \\
\text { clínico ra }\end{array}$ & $\sim$ & 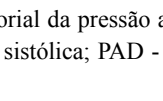 & 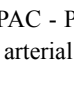 & $\begin{array}{l}\text { ial de consultório; HAS - Hipertensão arterial sistêmica; HVE - Hipertrofia ventricular } \\
\text { EAB - Efeito do avental branco; HAB - Hipertensão do avental branco; ECR - Estudo }\end{array}$ \\
\hline
\end{tabular}

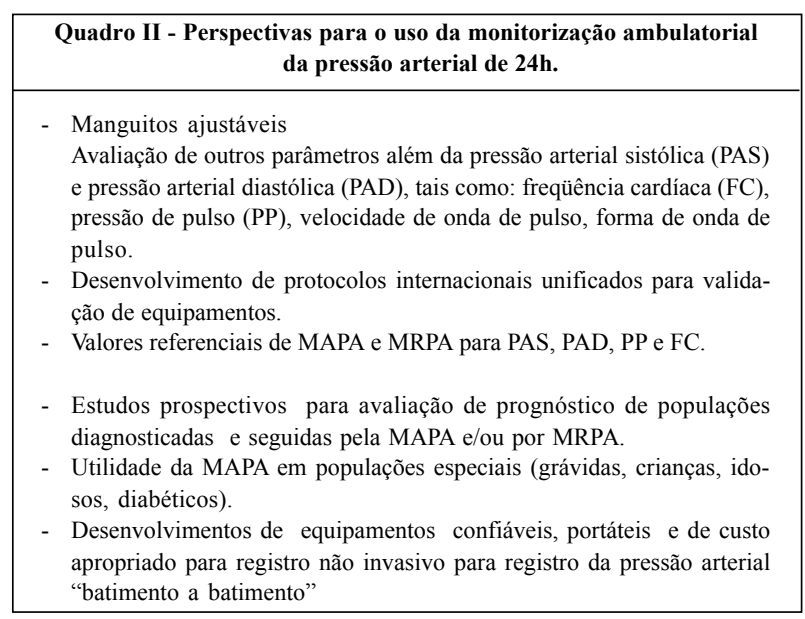

do método explorando-lhe os máximos benefícios a favor da compreensão da hipertensão e dos cuidados necessários para o seu tratamento.

A análise de outros parâmetros, que não somente os hoje classicamente avaliados; possibilidades de evoluções dos equipamentos e utilização em populações especiais são alguns dos pontos que, em próximo futuro, deverão ser incorporados à prática diária. O quadro II explicita futuras aplicações e possibilidades de uso da MAPA ${ }^{40}$.

Monitores mais baratos, confiáveis e confortáveis, ao lado de estudos demonstrando seu papel na redução de morbidade e mortalidade cardiovascular, quando utilizada para diagnóstico e seguimento no tratamento anti-hipertensivo, deverão ser o futuro próximo da monitorização ambulatorial da pressão arterial de $24 \mathrm{~h}$.

\section{Referências}

1. Riva-Rocci S. Um nuovo sfigmomanometro. Gazzeta Medica di Torino 1896;47: 981-1001.

2. Aiman D; Goldshine A. Blood pressure determinations by patients with essential hypertension. Am J Med Sci 1940; 200:465-74.

3. Veerman DP, de Blok K, Delamarre B, Van Montfrans GA. Office, nurse, basal and ambulatory blood pressure as predictors of hypertensive target organ damage in male and female patients. J Hum Hypertens 1996; 10: 9-15.

4. O'Brien E. Automated blood pressure measurement: State of the marketing in 1998 and the need for an international validation protocol for blood pressure measuring devices. Blood Press Monit 1998; 3: 205-11.

5. O'Brien E, Atkins N, Staessen J. State of the market: a review of ambulatory blood pressure monitoring devices. Hypertension 1995; 26:835-42.

6. Cuspidi C, Lonati L, Sampieri L, et al. Prevalence of target organ damage in treated hypertensive patients: different impact of clinic and ambulatory blood pressure control. J Hypertens 2000; 18:803-9.

7. Kain HK, HinmanAT, Sokolow M. Arterial blood pressure measurements with a protable recorder in hypertensive patients: variability and correlation with "casual" pressures. Circulation 1964;30:882-92.

8. HinmanAT, Engel BT, Bickford AF. Portable blood pressure recorder: accuracy and preliminary use in evaluating intra-daily variations in pressure. Am Heart J 1962;63:663.

9. The Sixth Report of the Joint National Committee (JNC) on Prevention, Detection, Evaluation, and Treatment of High Blood Pressure. Arch Intern Med 1997; 157:2413-46.

10. 1999 World Health Organization (WHO)-International Society of Hypertension (ISH) Guidelines for the Management of Hypertension. J Hypertens 1999; 17:151-83.

11. IV Diretrizes Brasileiras de Hipertensão Arterial. Rev Bras Hipertens 2002;4: no prelo.

12. Pickering TG. Future developments in ambulatory blood pressure monitoring in clinical practice. Blood Press Monit 2002;7:21-5.

13. Little P, Banett J, Barnsley L, Marjoram J, Fitzgeral'd-Baron A, Maut D. Comparison of agreement between different measurements of blood pressure in primary care and daytime ambulatory blood pressure. BMJ 2002;325:254-9.

14. McGrath B. Is white coat hypertension innocent ? Lancet 1996;348:630.

15. Muscholl M, Hense H-W; Brockel U; Doring A; Riegger G. Changes in left ventricular structure and function in patients with white-coat hypertension: cross sectional survey. BMJ 1998;317:565-70. 
16. Kattar RS, Semior R, Lahiri A. Cardiovascular outcome in white-coat versus sustained mild hypertension: 10 year follow-up study. Circulation 1998;98:1982-7.

17. Bidlingmeyer I, Burnier M, Bidlingmeyer M, Waeber B, Brunner HR. Isolated office hypertension: a prehypertensive state? J Hypertens 1996;14:327-32.

18. Mansoor GA, Mc Cabe EJ, White WB. Determinants of the white-coat effect in hypertensive subjects. J Hum Hypertens 1996; 10:87-92.

19. O'Brien E, Coats A, Owens P, et al. Use and interpretation of ambulatory blood pressure monitoring: recommendations of the British Hypertension Society. BMJ 2000; 320:1128-34.

20. Stergiou GS, Efstathiou SP, Argyraki CK, Gantzrou AP, Roussais LG. Clinical, home and ambulatory blood pressure: comparison and reproducibility. J Hypertens $2002 ; 20: 1897-3$

21. Pickering TG. Blood pressure measurement and detection of hypertension. Lancet 1994; 344:31-5.

22. Pickering TG. Ambulatory blood pressure monitoring. Curr Hypertens Repr 2000;2:558-64

23. Mancia G. Zanchetti A, Agabiti RE. Ffor the SAMPLE Study Group. Ambulatory blood pressure is superior to clinic blood pressure in predicting treatmentinduced regression of left ventricular hypertrophy. Circulation 1997;95: 1464-70.

24. Verdechia P. Prognostic. Value of ambulatory blood pressure: current evidence and clinical aplications. Hypertension 2000; 35:844-51

25. Perloff D, Sokolov M, Cowam RM. The prognostic value of ambulatory blood pressure. JAMA 1983;249:2792-8.

26. PerloffD, Sokolv M, Cowam RM, Juster RP. Prognostic value of ambulatory blood pressure measurements: further analyses. J Hypertens. 1989;7(suppl 3):S3-S10.

27. Okubo T, Imai Y, Tsujii I, et al. Predection of mortality blood pressure measurements: a pilot study in Ohasama. J Hypertens 1997;815:357-64.

28. Staessen JA, Trip L, Fagord R, et al. Predicting cardiovascular risk using conventional versus ambulatory blood pressure in older patients with systolic hypertension. The Systolic hypertension in Europe (Syst-Eur) trial investigators JAMA 1999;282:539-46.
29. O'Brein E, Sheridan J, O’Malley K. Dippers and non-dippers. Lancet 1988;2:397.

30. VerdechiaP,SchillaciG, Guerrieri M, etal.Circadiamblood pressure changes and left ventricular hypertrophy in essential hypertension. Circulation 1990;81: 528-36.

31. Bianchi S, Bigazzi R, Baldari G, Sgherri G, Campese VM. Diurnal variations of blood pressure and microalbuminuria in essential hypertension. Am J Hypertens 1994;7:23-4.

32. Shimada K, Kawamoto A, Matsubayashi K, Nishinaga M, Kimura S, Ozawa T. Diurnal blood pressure variations and silent cerebrovascular damoge in elderly hypertensive patients. J Hypertens 1992;10:875-8.

33. Parati G,Domidossi G, Albini F, Malospina D, Mancia G. Relationship of 24h mean and variability to severity of target organ damage in hypertension. $\mathrm{J}$ Hypertens 1987;5:93-8

34. Palatini P,Penzo M, RacioppaA, etal.Clinical relevance of night-time blood pressure and of day-time blood pressure variability. Arch Intern Med 1992;152: 1855-60.

35. Schillaci G, Verdechia P, Borgioni C, Cincci A, Porcelati C. Lack of association between blood pressure variability and left ventricular mass in essential hypertension. Am J Hypertens 1998;11:515-22.

36. Franklin SS, Kham SA, Wong ND, Larson MG, Levy D. Is pulse pressure useful in predicting risk of coronary heart disease? The Framingham Heart Study. Circulation 1999; 100:354-60

37. VerdechiaP,SchillaciG,BorgioniC, etal.Ambulatorypulse pressure: apatentpredictor of total cardiovascular risk in hypertension. Hypertension 1998;32: 983-8.

38. Staessen JA, Byttobyer G, Buntinix F, Celis H, O'Brein ET, Fagard R. Antihypertensive treatment based on conventional or ambulatory blood pressure measurements: a randomized controlled trial. JAMA 1997;278:1067-92.

39. Schrader J, Luders S, Zuchner C, Herbold M, Schrandt G. Pratice vs ambulatory blood pressure measurement under treatment with ramipril (PLUR Study): a randomized, prospective long-term study to evaluate the benefits of ABPM in patients ion antihypertensive treatment. J Hum Hypertens 2000; 14:435-40.

40. Parati G, Leeuw P, Illyes M, et al. In: 2001 Consensus Conderence on Ambulatory Blood Pressure Monitoring. Blood Press Monit 2002;7:83-7. 